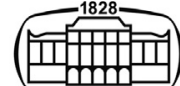

AKADÉMIAI KIADÓ

UNIVERSITY of DEBRECEN

\section{International Review of Applied Sciences and Engineering}

12 (2021) 2, 147-156

DOI:

$10.1556 / 1848.2021 .00214$

(c) 2021 The Author(s)

\section{ORIGINAL RESEARCH} PAPER

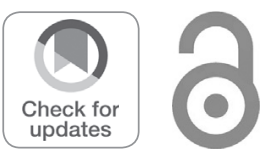

\title{
Novel approach in measurement instrument based on computer
}

\author{
Muaayed F. Al-Rawi ${ }^{1 *}$ (을 and Muhanned F. Al-Rawi ${ }^{2}$ \\ ${ }^{1}$ Computer Engineering Department, College of Engineering, Mustansiriyah University, \\ Baghdad, Iraq \\ ${ }^{2}$ Electrical Engineering Department, Faculty of Engineering, University of Ibb, Ibb, Yemen
}

Received: December 4, 2020 • Accepted: January 21, 2021

Published online: March 18, 2021

\begin{abstract}
Most applications in engineering use a data acquisition device hooked up to a personal computer for data processing. Finding less costly, easily accessible and reliable devices will make personal computer (PC) based data acquisition systems less difficult. A soundcard may be used as such a device for it is standard in almost every PC. It can also process any voltage signal within its limits. This paper proposes a way to enable the PC to be used as an oscilloscope. A voltage signal is acquired via the soundcard LINE IN port. The maximum and minimum input signal amplitude requirements for the soundcard are established to be $+1 \mathrm{~V}$ and $-1 \mathrm{~V}$ respectively. Based on these findings, hardware circuitry is designed to clip any high amplitude input signals to the range of $\pm 1 \mathrm{~V}$ while allowing low amplitude signals to go through to the soundcard unclipped. MATLAB is then employed to acquire, process and display the signal. The final output from MATLAB is compared with the original signal to determine accuracy of the designed oscilloscope. Analysis of the results obtained shows that the final oscilloscope designed enables the soundcard to process input signals with a high level of accuracy. The final design yields a hardware cost at a fraction of an iPod while providing an elegant user interface. This makes it suitable for college students, basement hackers and even professional engineers.
\end{abstract}

\section{KEYWORDS}

measurement instrument, computer, oscilloscope

\section{INTRODUCTION}

Engineering students who are studying the very abstract concepts of analog and digital signals rely greatly on the language of mathematics to describe the effect of circuits on signals. It would be much resourceful if they had pictures of these signals on an oscilloscope to complement the math. As a matter of fact, real data has a noise component which simulation generally lacks. As a direct consequence, a student testing a real circuit which has been constructed may be surprised to find that the output signals are very much different from those obtained from a simulation. Also, without the experience of connecting a circuit and making and correcting mistakes, the student will have little idea about what is wrong with a malfunctioning circuit and how to deal with the problem when faced with this situation on the job $[1,2]$.

However, the cost of laboratory equipment such as an oscilloscope is prohibitively expensive. Thus, the goal of this paper is to develop an inexpensive oscilloscope that can be used by the students and other people faced with this constraint. The oscilloscope is intended to provide the same laboratory experience as a commercially available one. In today's world, technology is advancing at a very fast pace and computer processing speeds are constantly being pushed to the limit. As people, especially in the developed world, acquire high speed computers, so old or second hand computers are made easily available to the third world at a cheap price. This has ushered in an era of personal computers and most students now own a computer, speed and performance notwithstanding. Since a soundcard is standard on most 
computers, it would be a noble idea to make use of the inherent digital signal processor to construct an oscilloscope $[3,4]$.

MATLAB is chosen as the software to develop the program to acquire signals from the soundcard and display them. The software also allows for the signal to be saved for future reference and analysis $[5,6]$.

The work in this paper is cost effective and offers reliable signal acquisition and processing capabilities at a fraction of a commercially available oscilloscope. The resulting program is made independent of MATLAB by making it into an executable file using the deployment feature of MATLAB 7.6. This means that the user will not have to purchase MATLAB to run this program. The oscilloscope designed is thus ideal for college students, hobbyists and professional engineers alike.

\section{DESIGN PROCEDURE}

\subsection{Attenuator}

As the name suggests, this circuit is designed to attenuate the input voltage signal to a level that can be observed on the screen. Since the clipping circuit is designed to clip all voltages to the range of $\pm 1 \mathrm{~V}$, it is instructive to reduce the input voltage amplitude to the range of $\pm 1 \mathrm{~V}$, otherwise the signal will appear as clipped on the display screen thus hiding other signal details. The attenuation circuit provides a way of avoiding such a nasty occurrence.

The attenuation circuit also doubles up as high impedance pre-scalar. Commercial oscilloscopes have input impedances of the order of $1 \mathrm{M} \Omega$ thus they do not load the circuit being probed. To ensure that the designed oscilloscope has high input impedance, huge resistors are used in this circuit of the order of $\Omega \mathrm{M}$. This will enable it compete favorably with commercially available ones.

There is a knob on the front panel for the user to select the amount of attenuation needed. The circuit attenuates high amplitude voltages but does not attenuate the low amplitude voltages. This is a desirable feature since the quantization potential of a small signal will not be reduced thus enhancing its resolution.

The circuit is implemented as shown in Fig. 1, using a double-pole six-position rotary switch to select the different voltage divider positions. However, during testing it is discovered that the true resistance of the large resistors had frequency dependence. This is due to the resistor leads acting as inductors at high frequencies.

\subsection{The probe}

The oscilloscope is mainly used to display voltage signals. This means that a voltage probe is needed to achieve the same. Initially, the plan is to design a new probe from scratch to meet the specifications. This would help drive down the cost of the entire design.

The general consensus is that no single probe can achieve all the probing requirements of every circuit. The goal thus is

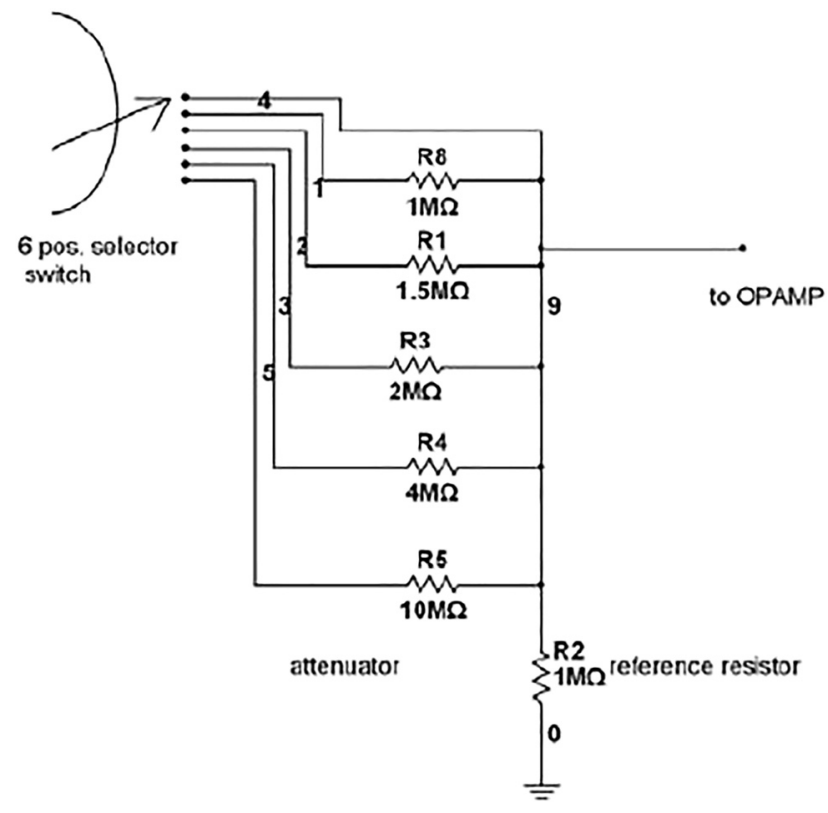

Fig. 1. Attenuator circuit

to select a probe that delivers the best representation of a signal to an oscilloscope. In choosing the probe, four fundamental signal source issues are considered, that is: signal type, signal frequency content, source impedance and the physical attributes of the test point.

\subsection{Soundcard interface}

The entire signal conditioning box had to interface to the PC via the soundcard. Since this represents data or signal input to the soundcard, it means the LINE IN and the MIC IN ports are the only input ports available for use. However, the microphone input offers better sensitivity but there is a dc voltage in this port for powering microphones. Since the user may interface the signal conditioning box to the PC via this MIC IN port, a capacitor in the signal paths had to be employed to block this voltage. Under normal circumstances, the LINE IN port should be used for signal input. The MIC IN port could be used when high sensitivity is required.

2.3.1. RCA to RCA cable. The decision for the connection from the signal conditioning circuit to the PC is a particularly challenging one. Two options are available:

1. Use a shielded coaxial cable with BNC connectors on both sides, i.e. male connector on the side of the box and a female BNC connector on the PC side.

2. Use Radio Corporation of America (RCA) to RCA cable.

The latter is chosen since the former is more expensive to implement. The cost of a coaxial cable per meter is prohibiting considering the fact that the cost of the design had to be kept low. Also, it would mean purchasing Bayonet Neill-Concelman (BNC) connectors and soldering them to the cable thus increasing the design time. 
Luckily, an off the shelf RCA to RCA connector is available at a cheap price and provided satisfactory performance. Another inherent advantage of this cable is that it is shielded thus guaranteeing noise immunity. This cable can also support two channel operation, however since the hardware currently supports only single channel operation, one path of the cable is used.

2.3.2. Stereo to 2-RCA connector. Both the LINE IN and the MIC IN ports of the soundcard are stereo in nature which means they have two channels. RCA to RCA cable is used to pipe the signal from the signal conditioning box to the soundcard. A way of interconnecting the two - a male RCA connector and a stereo input port had to be figured out. A cheap solution is the stereo to 2-RCA connector that effectively splits the two channels of the stereo input port into its constituent channels and makes them available through two female RCA connectors.

\subsection{Voltage over-load protection circuit}

All signal inputs to a soundcard must be restricted to $\pm 1 \mathrm{~V}$. Failure to observe this will lead to serious damage to the soundcard circuitry. The voltage protection circuit, or voltage clipper, is a simple circuit designed to protect the soundcard. It forces the high amplitude input signal to a window of $\pm 1 \mathrm{~V}$.

In the initial design, zener diodes are used to clip the voltage amplitude of the input signal to the required level. The zener diodes are operated in their breakdown region. They are supposed to have a breakdown voltage of $1 \mathrm{~V}$. However, it is discovered that zener diodes have a lot of noise in their breakdown region. Since the oscilloscope is expected to measure low level voltage signals, this feature is undesirable since it would be hard to differentiate between the actual signal and the noise due to breakdown.

The best alternative is the schottky diode. They have rapid response to high frequency signals. The schottky diodes are arranged such that the forward voltage drop is $1 \mathrm{~V}$ for both the positive and negative going signals. A resistor is placed in series with the diode arrangement so as to limit current flow. A voltage follower is implemented between the attenuator circuit and the voltage overload protection circuit. This is to prevent the clipper circuit from loading the attenuator circuit which would have the adverse effect of giving erroneous voltage division values. Voltage follower op-amp has an ideal input impedance of infinity but zero ideal output impedance. Thus, they are a good candidate for isolating the two modules of the circuit.

The purpose of any engineering design work is to meet the specifications required. The methodologies and components used may vary but the end ultimately justifies the means. Consequently, schottky diodes could not be found locally and hence the design had to be changed to allow use of locally available components. The final design thus used silicon signal diodes connected in series to give a forward voltage drop of $1 \mathrm{~V}$ at room temperature. Signal diodes were chosen because of their high switching speeds compared to ordinary diodes.

\subsection{Power supply circuit}

The PC oscilloscope requires power supply to function. This is because the op-amp used to isolate the attenuator circuit from the clipping circuit needs a power supply of about \pm 10 $\mathrm{V}$.

The circuit takes power from two commercially available $9 \mathrm{~V}$ batteries and produces two voltage levels of $+9 \mathrm{~V}$ and -9 $\mathrm{V}$ for powering the op-amp. The two batteries are connected in series. The junction of the two batteries is grounded to provide the reference for the voltages. The circuit of this module is shown in Fig. 2.

One of the outputs is then connected to $V_{c c}$, while the other to $\mathrm{V}_{\mathrm{ee}}$. A capacitor is connected across both outputs to ensure a smooth output.

\subsection{PC host software}

It is important that the PC software resembles a real oscilloscope as closely as possible. Should the PC software differ substantially from the commercially available oscilloscope, then the software has a learning curve and improvements

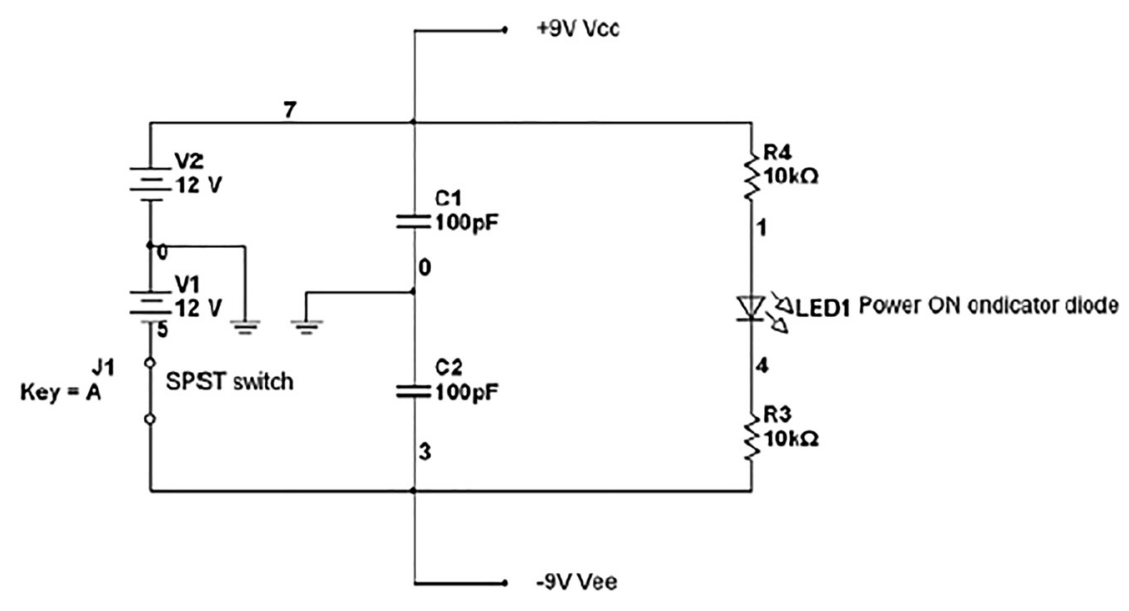

Fig. 2. Power supply circuit 
can be made over time. However, the author wishes to submit that such a scenario is not desirable especially when trying to provide an alternative product. On the other hand, there are some things one can do on the PC that cannot be done on a physical oscilloscope. This include saving a signal and even displaying the spectrum of the signal. Such are expected to be the greatest selling points of the design.

Two development platforms are available that could perform the task of reading the PC soundcard quite comfortably. These are MATLAB and National Instrument's LABVIEW. The latter, though simpler to implement using virtual instruments, is not chosen for this task. This is largely because of availability. MATLAB development environment is readily available with full functionality but LABVIEW proved hard to get. There are other inherent advantages in using MATLAB as opposed to other software. MATLAB is platform independent; this means that it can be deployed on any operating system including WINDOWS and LINUX. Additionally, MATLAB runtime environment need not be installed on the client machine for the software to function.

2.6.1. Design methodology. The design of the host software is divided into two major categories

1. Driver and application software design

2. User interface (UI) design

2.6.1.1. Driver and application software design. Due to limited development time, two critical decisions are made:

i. Software development would occur in parallel with hardware development.

ii. Both application and driver software would be lumped together and developed as a single entity.

Development of this part of software is arguably the hardest. First a software algorithm of the software functionality is developed. The algorithm is as listed below:
1. Create user interface - the user interface for the oscilloscope is first created including the axes and the entire user controls.

2. Create a device object - a device object is then created using the analog Input, creation function. Device objects are the basic toolbox elements used to access the hardware device.

3. Add channels - after a device object is created, channels are added to it. Channels are added to analog input and analog output objects, while lines are added to digital I/O objects. Channels and lines are the basic hardware device elements used to acquire or output data. Since two channel operation is envisaged, two analog channels are added to the object.

4. Configure properties - to establish the device object behavior, values are assigned to properties using the set function or dot notation. Set function notation is used due to its simplicity, some of the properties configured include sample rate, samples per trigger and trigger mode.

5. Start acquisition of data - to acquire data, the device object is started with the start function. Acquisition occurs in the background, while MATLAB continues executing. Other MATLAB commands can be executed while the acquisition is occurring.

6. Wait for the acquisition to complete - the wait function is used to pause MATLAB until the acquisition is complete.

7. Extract the acquired data (analog input only) - after data is acquired, it is extracted from the engine using the GetData function.

8. Plot the data - the data is displayed on the axes.

9. Clean up - when the device object is no longer needed, it is removed from memory using the delete function.

2.6.1.2. User interface design. Once the driver and application software is designed, the user interface had to be put in place. This will allow users to interact with the

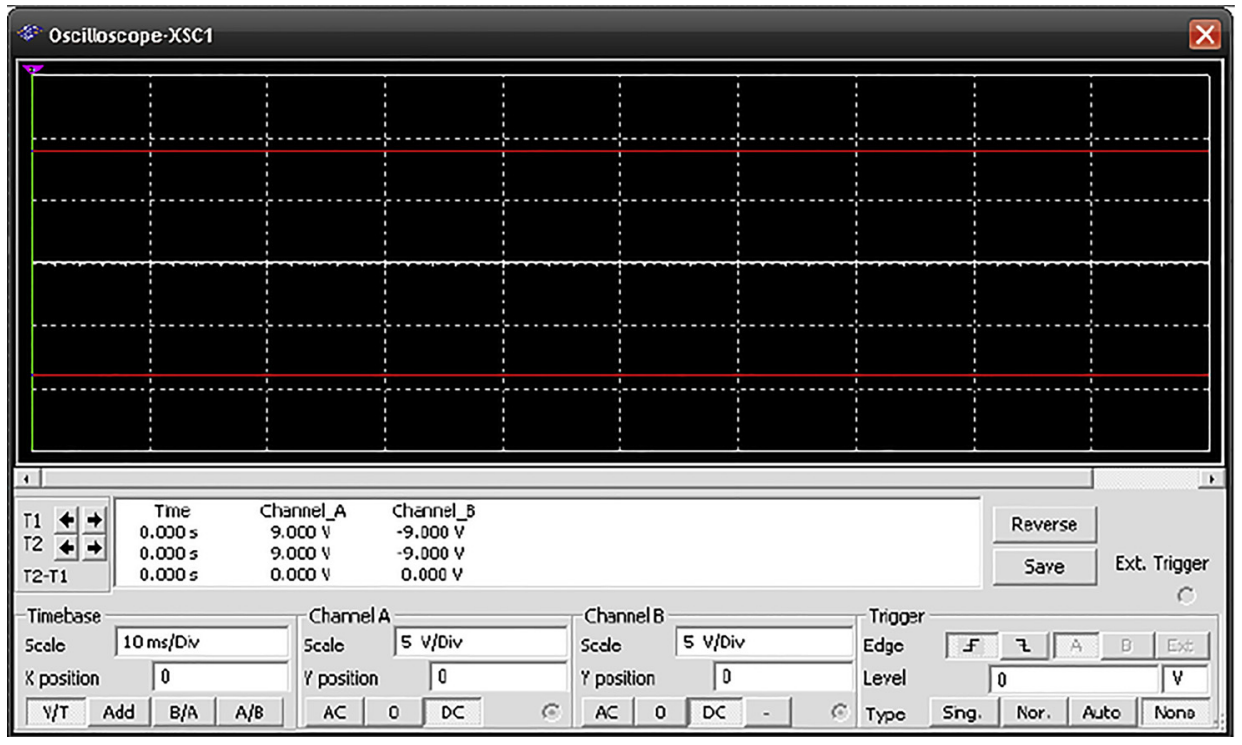

Fig. 3. Power supply circuit output with time 
oscilloscope by setting various values on the user control buttons and textboxes. Less coding is involved at this juncture since all that had to be done is to call the functions already created earlier to respond to mouse events.
MATLAB has a user design instrument called Graphic User Interface Design Environment (GUIDE). Initially this tool is used for the design of the user interface; however, it proved difficult especially in linking the various callbacks to

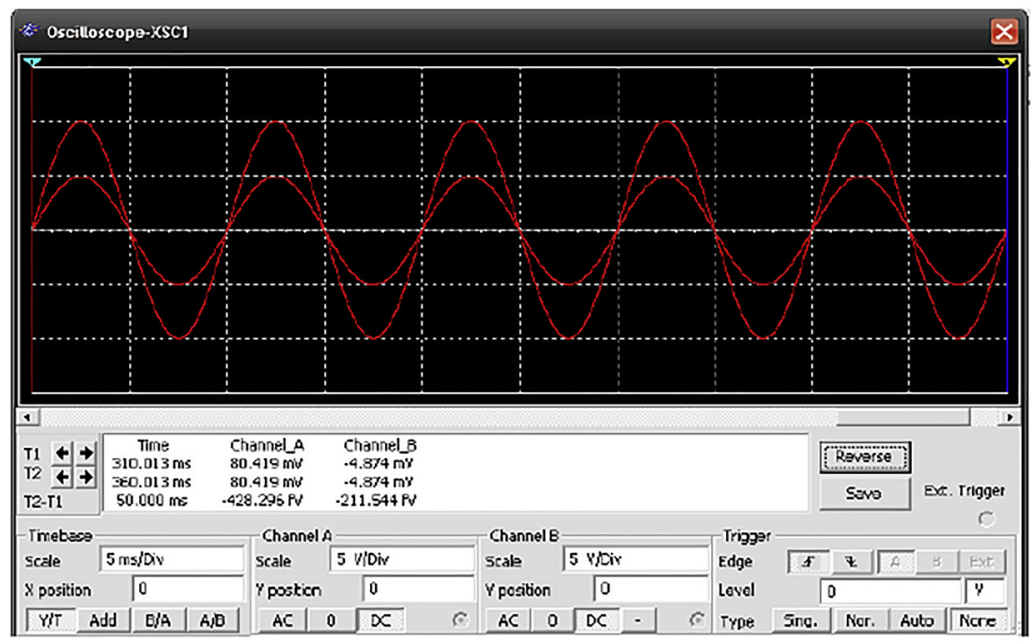

a) Input frequency: $100 \mathrm{~Hz}$, input voltage: $20 \mathrm{Vpp}$, divider output: $10 \mathrm{Vpp}$, Scale: 1:2

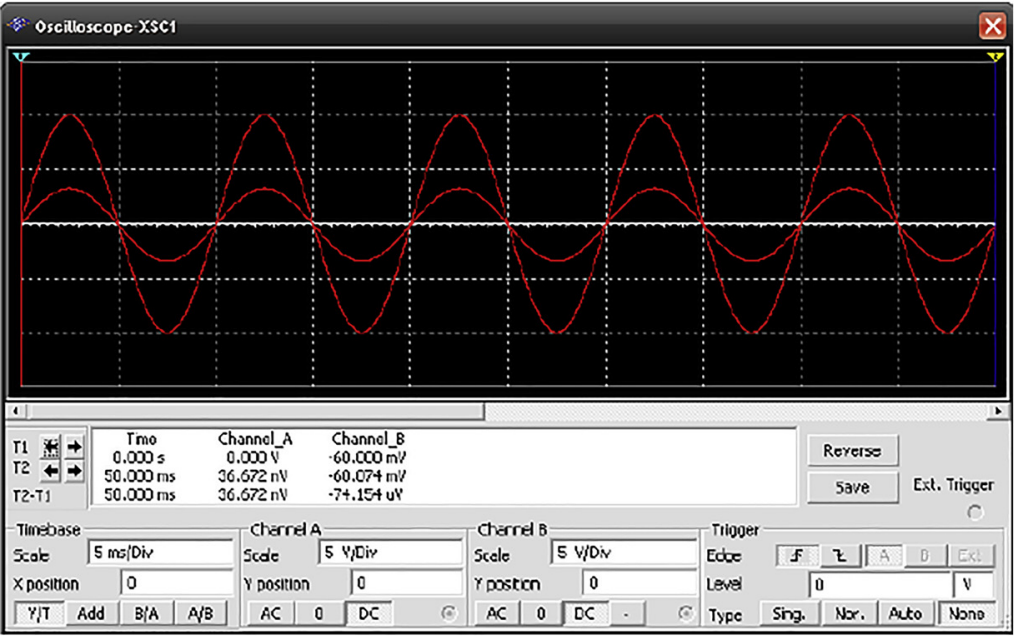

b) Input frequency: $100 \mathrm{~Hz}$, input voltage: $20 \mathrm{Vpp}$, divider output: $6 \mathrm{Vpp}$, Scale: 1:3

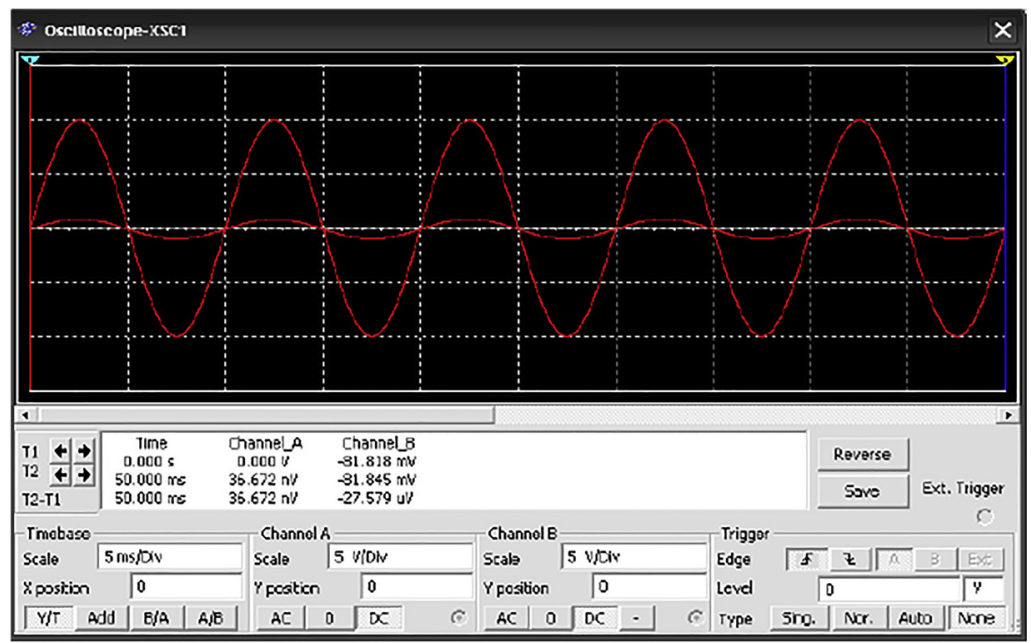

c) Input frequency: $100 \mathrm{~Hz}$, input voltage: $20 \mathrm{Vpp}$, divider output: $2 \mathrm{Vpp}$, Scale: 1:11

Fig. 4. Voltage division tests for selected attenuation levels with time 
the desired functions. A decision is thus made to generate the UI programmatically. This proves a wise decision since design progresses are quite fast with few errors.

In the design of the UI, it is imperative that it resembles an oscilloscope as closely as possible as well as provides aesthetic appeal to the user. The UI design followed four basic principles abbreviated as CRAP. These are Contrast, Repetition, Alignment and Proximity:

a. Contrast. Contrast refers to any difference of size, shape, or color used to distinguish between the various UI elements and user control components. The color red is chosen for the axes on a black background. These are bright colors that allow for the signal, which is plotted in yellow color to be discerned clearly.

b. Repetition. The size and typeface for the various text labels is uniform for the UI. Such repetition of design elements pulls the UI together into a cohesive whole and improves readability.

c. Alignment. Alignment is crucial for the cohesive appearance of the UI. All the text used on the UI is aligned to a common vertical baseline, otherwise, unaligned text floats mysteriously forcing the user to figure out its relation to the rest of the UI.

d. Proximity. Pieces of information that are supposed to complement each other should be near each other e.g.: The start and stop push buttons are placed together since their actions are complimentary. The axis is isolated in the middle of the UI to catch the attention of the eye.

2.6.2. Signal windowing. Some of the windows available in MATLAB include:
- Blackman window

- Hamming window

- Rectangular window

- Triangular (Bartlett) window

The rectangular window is good for separating signals that are close in frequency and have similar amplitudes. The hamming window is widely used in the area of speech signal processing. The rectangular window is thus most suitable for this paper and is thus chosen. The rectangular window is the default window for MATLAB. This means that it did not have to be explicitly defined in the code written, in fact, in MATLAB DSP block, a rectangular window is equivalent to no window at all.

\section{VERIFICATION}

In order to verify that the original designs function correctly, the function of a few key modules has to be verified first. These include the power supply circuit, the attenuator and the voltage clipping circuit. Based on the results of these tests, the kind of functionality that they provide would be known. After some of the preliminary testing is completed, it would be easier to determine if the design meets the basic functionality.

\subsection{Power supply circuit}

Engineers grapple with acquisition of various signals in the field. This means that a lighter and portable design for our oscilloscope will have an added advantage over the commercially available ones whose weight is quite overbearing thus discouraging portability. To achieve this, it is instructive for the signal conditioning box to have its own

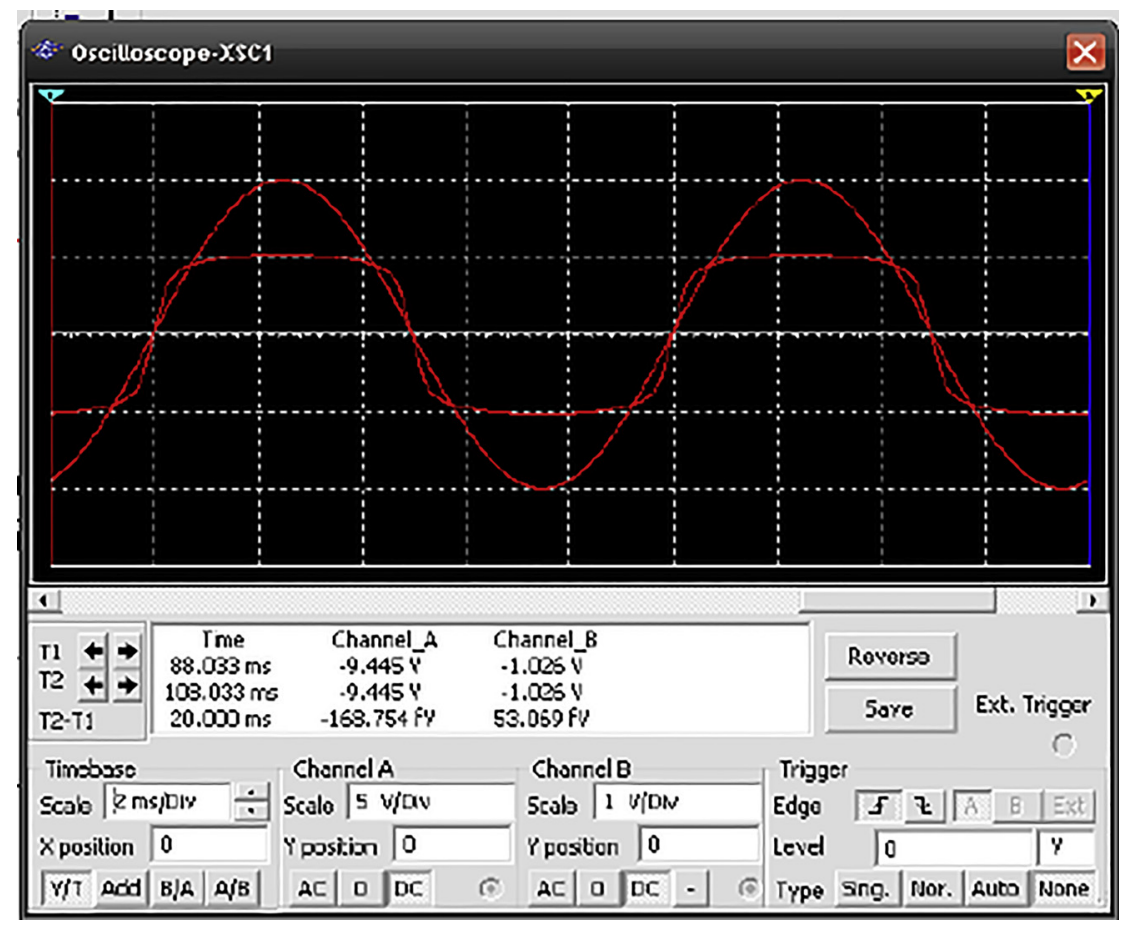

Fig. 5. Voltage clipping with time 


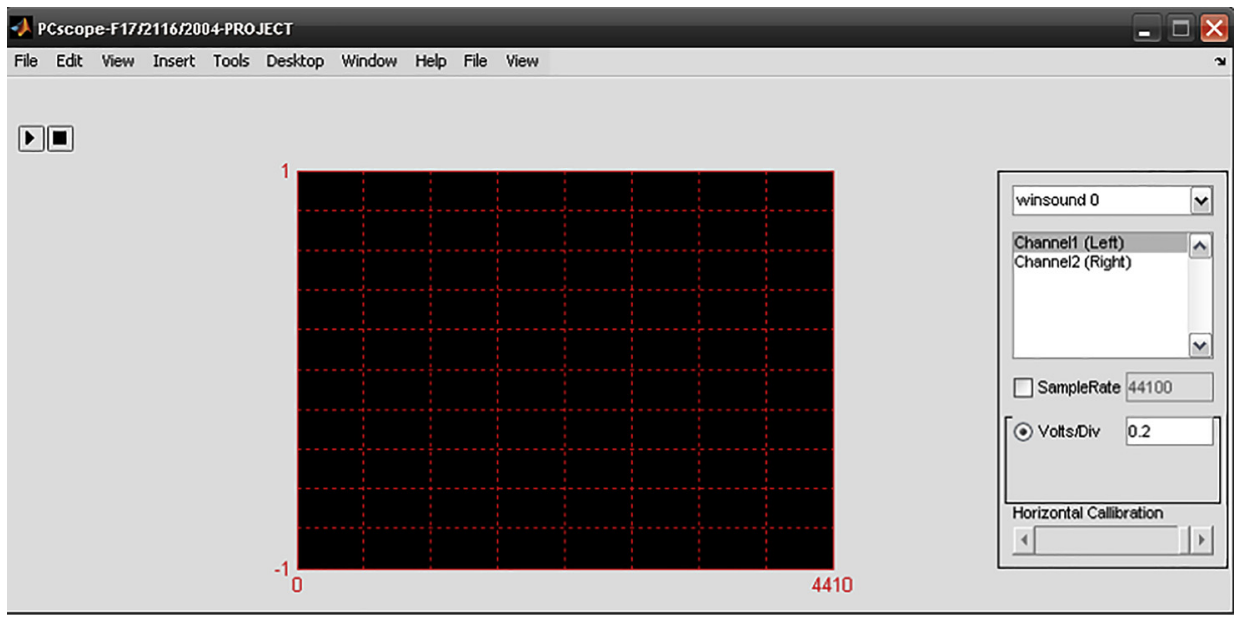

Fig. 6. The designed UI

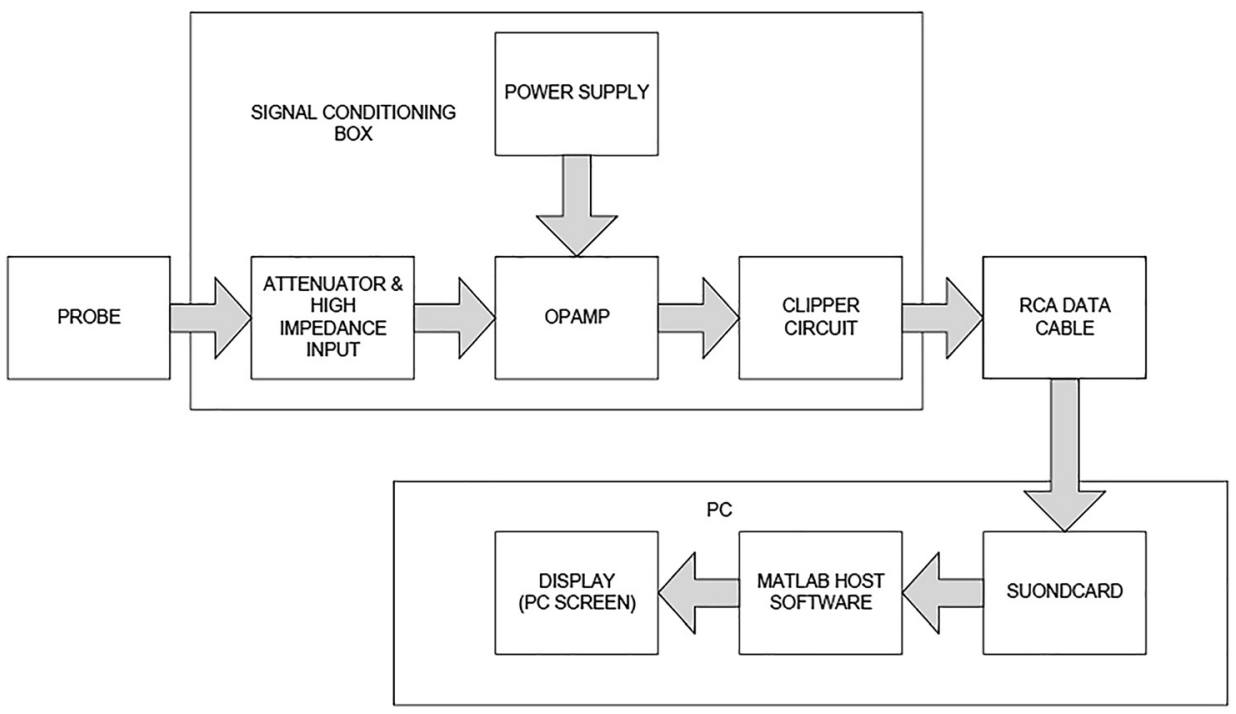

Fig. 7. System block diagram

power supply. The circuit shown in Fig. 2 is set up and the output is measured using an oscilloscope. The results are shown in Fig. 3. The results prove that the circuit is capable of splitting the $18 \mathrm{~V} \mathrm{DC}$ voltage supply into two $\pm 9 \mathrm{~V}$ rails for powering the op-amp.

\subsection{Attenuator circuit}

Voltage attenuation is tested post-production modularly and also in conjunction with the voltage clamp circuit. As mentioned earlier, the attenuator circuit doubles up as high input impedance for the oscilloscope to avoid loading of the test circuit. The circuit of Fig. 1 is set up in the laboratory. Signals of varying amplitudes are then connected to the input of the circuit and the output observed on an oscilloscope. The attenuator circuit is then shifted through all the attenuation levels using the six-position switch and the effect on the input waveform also observed on the

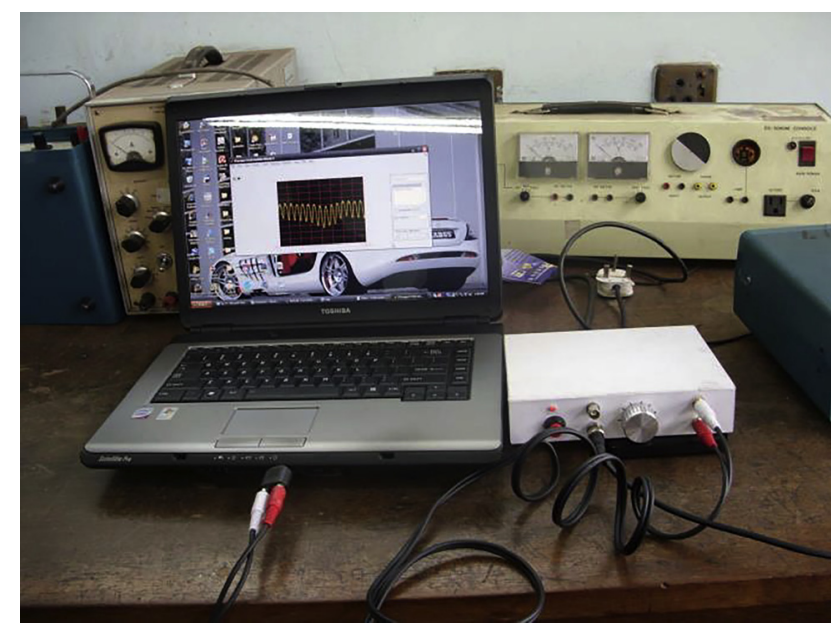

Fig. 8. System prototype 
oscilloscope to determine if different attenuation levels could be attained.

The results show that the attenuation circuit performs as expected reducing the peak-to-peak voltage of the

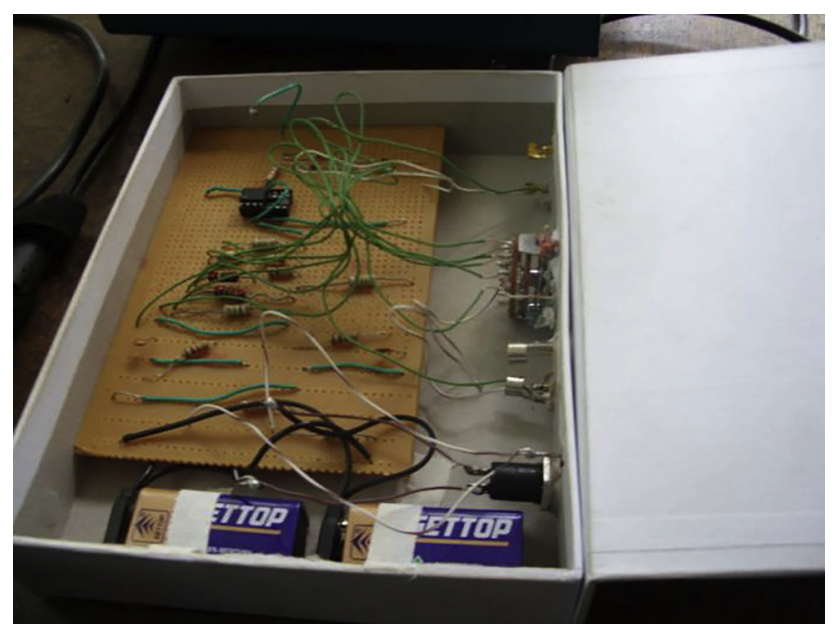

Fig. 9. Inside the signal conditioning box waveforms as expected. The results are documented in Fig. 4. An additional concern, which is not evaluated since bandwidth is less than $1 \mathrm{MHz}$, is the skin effect, which describes the phenomenon where, at frequencies of and above $1 \mathrm{MHz}$, electrons begin to avoid traveling down the center of the conductor, decreasing the conducting surface area and creating additional resistance within the conductor. This would add additional non-linear division effects to the circuitry.

\subsection{The probe}

Testing of the probe is relatively easy, the selected probe is first connected to a laboratory oscilloscope and a test circuit probed. It is established that no loading of the test circuit is encountered since expected signals are observed.

\subsection{Soundcard interface}

Testing of the soundcard interface is a relatively simple issue. First a signal had to be piped from a conventional laboratory signal generator to the soundcard and

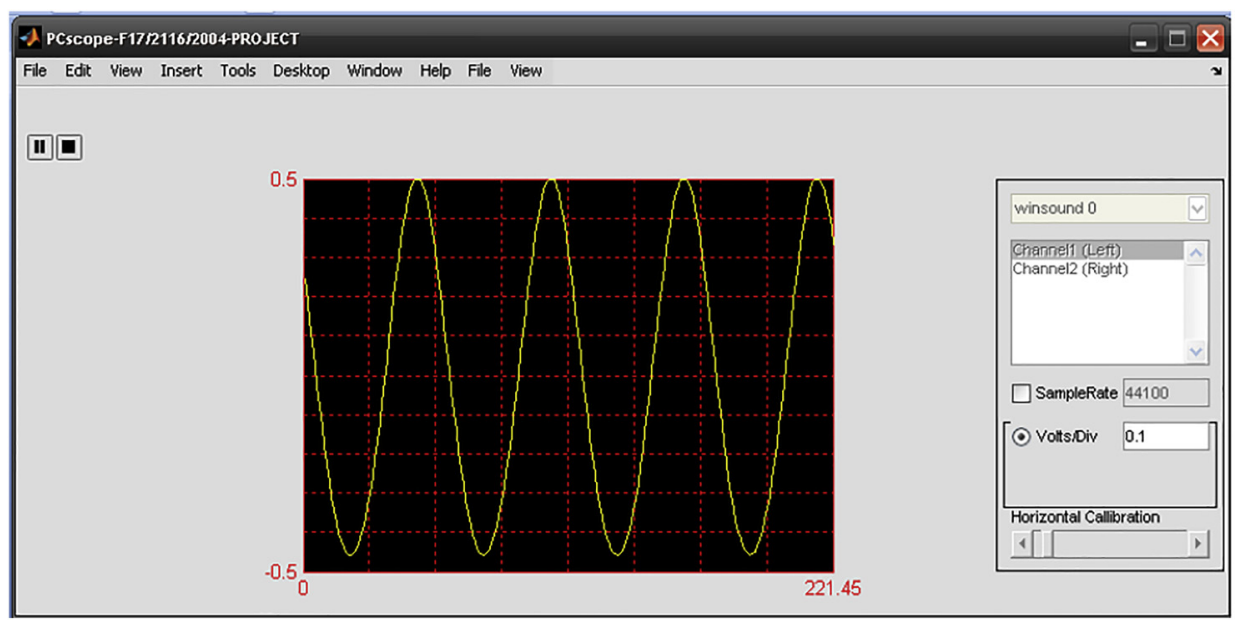

a) Sine wave at $100 \mathrm{~Hz}$

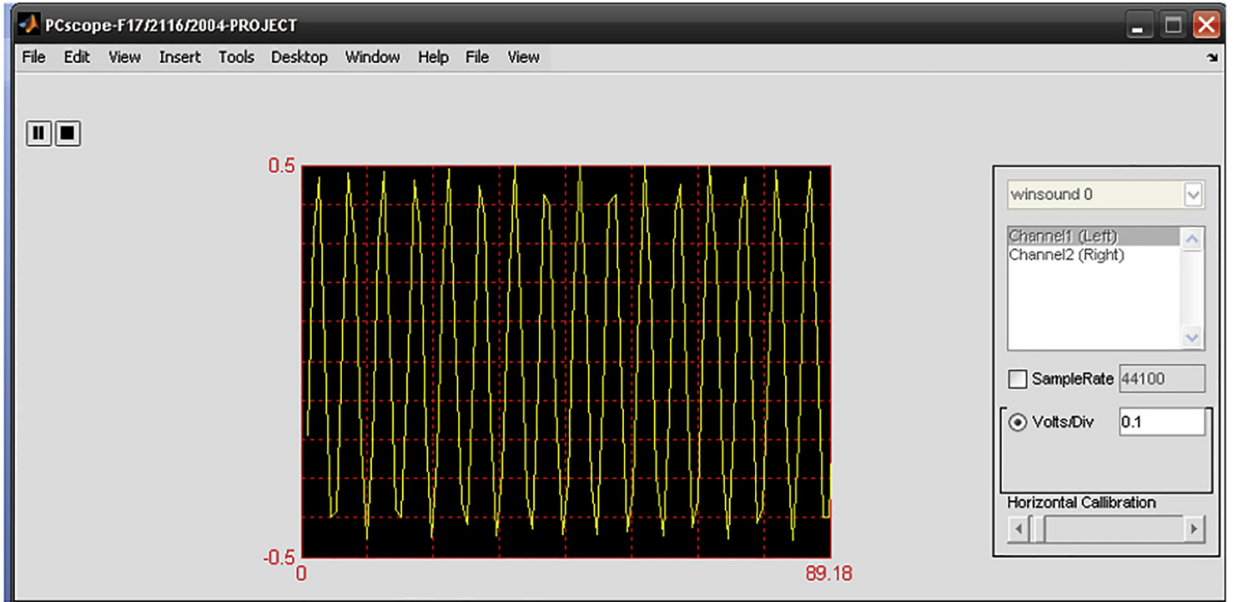

b) Sine wave at $1 \mathrm{KHz}$

Fig. 10. Sine waves as acquired by the PC scope in real time 
displayed by the host software. This end is achieved quite successfully and both sine and square waves are acquired from the signal generator. However, great care is taken to ensure the input signal does not go above $2 \mathrm{~V}$ peak-topeak since the voltage overload protection circuit had not been connected yet. After getting the basic signal acquisition working, the RCA cable with a stereo to RCA connector hooked to one end is then connected directly to the signal conditioning box to work in tandem with the signal conditioning circuit.

\subsection{Voltage overload protection}

The voltage overload protection, or voltage clipper, is tested both modularly and in conjunction with the attenuator circuit and other circuit modules as a complete circuit. The results are as shown in Fig. 5. The results of the tests on the circuit show that the circuit does in fact clip voltages of up to $20 \mathrm{~V}$ peak-to-peak to a $2 \mathrm{~V}$ peak-topeak threshold. This is a safe input voltage for the soundcard and the internal circuitry of the same is protected from overload.

\subsection{PC host software}

The host software is particularly difficult to test because the software development had to occur in parallel with the hardware development. This means that most of the time there is no way to stream data into the software. However, after some reasonable time of debugging the software, it is able to display the waveforms and the user interface control components are able to respond as expected. Figure 6 shows the user interface as designed.

\subsection{Overall circuit testing}

After the design is completed, all the modules are assembled together and various kinds of waveforms acquired in various conditions. Figure 7 shows the system block diagram; Figure 8 shows system prototype, and Fig. 9 shows inside the signal conditioning box. The oscilloscope is found to acquire signals with a high level of accuracy and minimal distortion. Figure 10 shows a sine waves in real time, while Fig. 11 shows square waves acquired by the oscilloscope. The acquired waves are compared with the

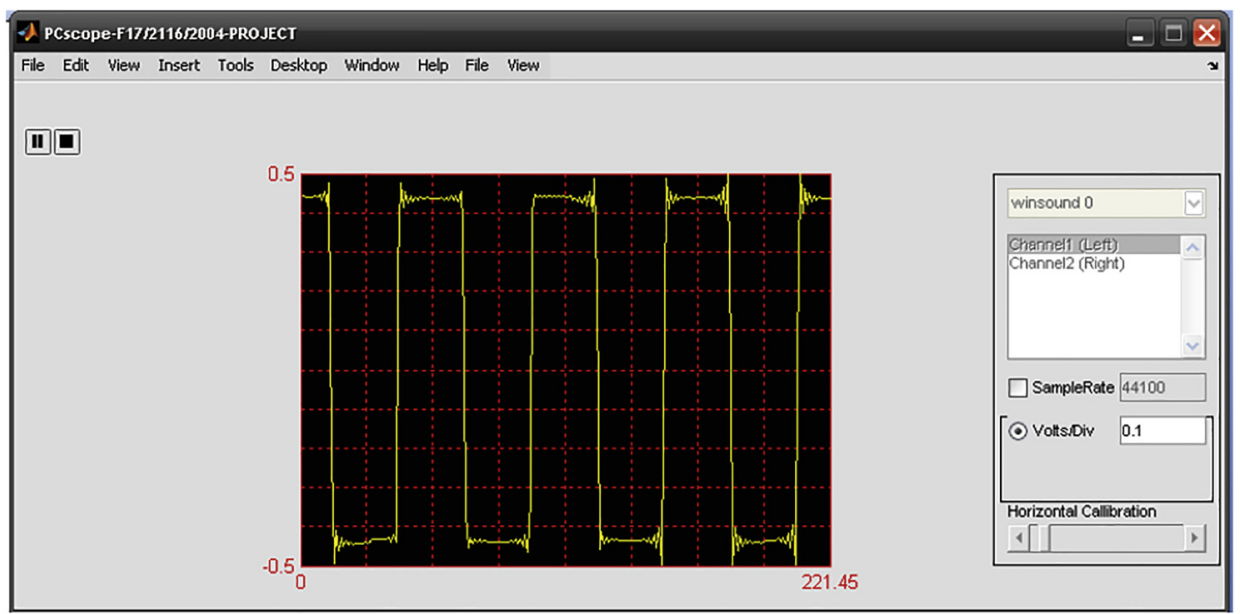

a) Square wave at $100 \mathrm{~Hz}$

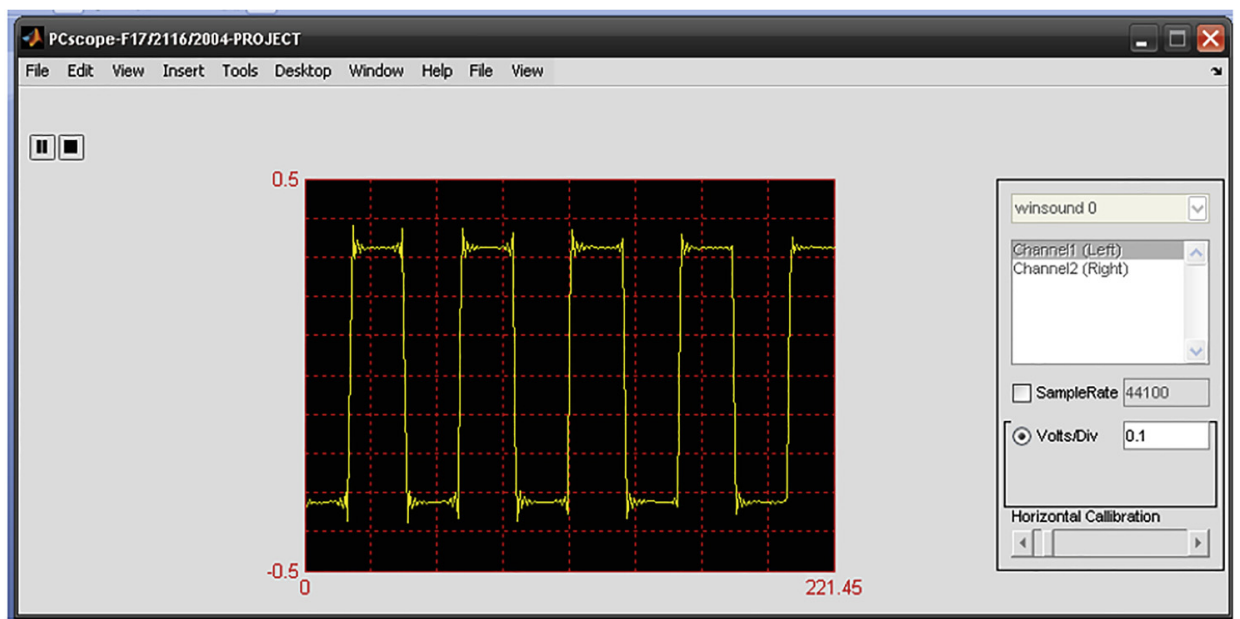

a) Square wave at $100 \mathrm{~Hz}$

Fig. 11. Real time square waves as acquired by the PC scope 
original ones as observed on a laboratory oscilloscope and they are found to resemble.

\section{CONCLUSION}

The conclusion of this paper provided a sense of accomplishment and general contentment. However, there are some concerns for the future. The ability and general performance of the oscilloscope in relation to the scope of the entire paper is satisfactory. As far as the hardware is concerned, the current design has pros and cons. The hardware's ability to protect the soundcard and attenuate high amplitude signals while letting low amplitude signals pass without attenuation is an achievement. The hardware interface works well and is fairly straightforward, but there are areas of design that need to be addressed before the system is considered reliable.

A larger uncertainty of the final design comes from the fact that the square waves acquired exhibit Gibbs phenomenon. This can be attributed to the discontinuities (vertical edges) in a square wave. Discontinuities are events which occur in zero time. An ideal square wave changes from maximum positive value to a maximum negative value in zero time. There is no such thing in the real world. By Fourier series, any periodic waveform can be synthesized using sinusoidal waves. Usually, using more sinusoidal waves produces better results. To approximate a discontinuity such as the one present on an ideal square wave, it requires a large number of harmonics. This translates to a high bandwidth (BW). Unfortunately, most soundcards have a maximum sampling rate of $44,100 \mathrm{~Hz}$ thus limiting the BW to a Nyquist frequency of $11,050 \mathrm{~Hz}$ ideally. Thus, to get a better approximation of a square wave, a high quality soundcard with a high sample rate specification is required.

\section{ACKNOWLEDGMENT}

The author would like to thank Mustansiriyah University (www.uomustansiriyah.edu.iq) Baghdad - Iraq for its support in the present work.

\section{REFERENCES}

[1] C. Bhunia, S. Giri, S. Kar, S. Haldar, and P. Purkait, "A low-cost PC-based virtual oscilloscope," IEEE Transaction on Edu., vol. 47, no. 2, pp. 295-9, 2004.

[2] S. Verma, R. Baghmar, and S. Agrawal, "PC based oscilloscope," Int. J. Eng. Res. Technol., vol. 2, no. 10, pp. 2083-94, 2013.

[3] D. Patil and V. Umale, "Oscilloscope: A PC-based real time oscilloscope," Int. J. Eng. Res. Appl., vol. 4, no. 4, pp. 253-8, 2014.

[4] S. Garg, "Designing a PC based oscilloscope using Arduino," Int. J. Electr. Electron. Res., vol. 3, no. 2, pp. 253-5, 2015.

[5] F. Russo and F. Travain, "Programming PC-based multi-function oscilloscopes: A user-friendly approach to rapid prototyping of spectral analysis code," Int. J. Instrum. Meas., vol. 2, pp. 1-10, 2017.

[6] R. Tare, R. Bhonde, and S. Gupta, "PC based oscilloscope using Arduino," International Conference on Emanations in Mordern Engineering Science \& Management, India, 2018. 